\title{
INTEGRABLE BOUNDARY INTERACTIONS FOR RUIJSENAARS' DIFFERENCE TODA CHAIN
}

\author{
J.F. VAN DIEJEN AND E. EMSIZ
}

\begin{abstract}
We endow Ruijsenaars' open difference Toda chain with a onesided boundary interaction of Askey-Wilson type and diagonalize the quantum Hamiltonian by means of deformed hyperoctahedral $q$-Whittaker functions that arise as a $t=0$ degeneration of the Macdonald-Koornwinder multivariate Askey-Wilson polynomials. This immediately entails the quantum integrability, the bispectral dual system, and the $n$-particle scattering operator for the chain in question.
\end{abstract}

\section{Introduction}

It is well-known that the open and closed Toda chains may be viewed as limits of the hyperbolic and elliptic Calogero-Moser-Sutherland particle systems, respectively [St, R1, I, R2. More general integrable open Toda chains with boundary interactions involving potentials of Morse type [Ko, GW, Sk1] and of PöschlTeller type [I, KJC] are recovered similarly as degenerations of the OlshanetskyPerelomov-Inozemtsev generalized Calogero-Moser-Sutherland systems with hyperoctahedral symmetry [I, O, Sh, GLO2]. Moreover, such limiting relations turn out to persist at the level of the Ruijsenaars-Schneider particle systems and Ruijsenaars' difference (a.k.a. relativistic) Toda chains [R1, R2, R3, E, GLO1, HR, BC] as well as their hyperoctahedral counterparts $[\mathrm{D} 2, \mathrm{C}$. Specifically, in the hyperoctahedral case one recovers in this manner generalizations of Ruijsenaars' open relativistic Toda chain with boundary interactions that were studied at the level of classical mechanics in Refs. Su1, D1, Su2 and at the level of quantum mechanics in Refs. $\mathrm{KT}, \mathrm{D} 2, \mathrm{E}, \mathrm{S}, \mathrm{C}$.

In the present work we consider the Hamiltonian of such an open difference Toda chain endowed with a one-sided four-parameter boundary interaction of AskeyWilson type. Upon diagonalizing the quantum Hamiltonian in question by means of deformed hyperoctahedral $q$-Whittaker functions that arise as a $t=0$ degeneration of the Macdonald-Koornwinder polynomials $[\mathrm{K}, \mathrm{M}$, the quantum integrability, the bispectral dual system, and the $n$-particle scattering operator are deduced. For special values of the Askey-Wilson parameters, our chain amounts to a difference counterpart of the $D_{n}$-type and the $A_{n-1}$-type quantum Toda chains with one-sided boundary potentials of Pöschl-Teller and Morse type, respectively.

Date: May 2014.

2000 Mathematics Subject Classification. 81Q80, 81R12, 81U15, 33D52.

Key words and phrases. difference Toda chain, boundary interactions, quantum integrability, bispectral duality, $n$-particle scattering, hyperoctahedral $q$-Whittaker functions.

This work was supported in part by the Fondo Nacional de Desarrollo Cientifico y Tecnológico (FONDECYT) Grants \# 1130226 and \# 1141114. 
The presentation is structured as follows. After introducing our difference Toda chain in Section 2 and defining the deformed hyperoctahedral $q$-Whittaker functions in Section 3, the diagonalization of the Hamiltonian is carried out in Section 4 by identifying the corresponding eigenvalue equation with the $t \rightarrow 0$ degeneration of a well-known Pieri formula for the Macdonald-Koornwinder polynomials [D3, M]. The quantum integrals and the bispectral dual system are then discussed in Sections 5 and 6 respectively. In Section 7 analogous results for a difference counterpart of the quantum Toda chain with one-sided boundary potentials of Morse type are obtained by letting one of the boundary parameters tend to zero (which corresponds to a transition from Askey-Wilson polynomials to continuous dual $q$-Hahn polynomials [KLS]). We close in Section 8 with an explicit description of the $n$-particle scattering operator that relies on a stationary-phase analysis that was performed in

Refs. R4, D4. Some useful properties of the Macdonald-Koornwinder multivariate Askey-Wilson polynomials have been collected in a separate appendix at the end.

\section{Difference Toda Chain With ONE-Sided Boundary INTERACTION OF ASKey-WILSON TYPE}

Formally, the Hamiltonian of our difference Toda chain is given by the difference operator [D2]:

$$
\begin{aligned}
H:= & T_{1}+\sum_{j=2}^{n-1}\left(1-q^{x_{j-1}-x_{j}}\right) T_{j} \\
& +\sum_{j=1}^{n-2}\left(1-q^{x_{j}-x_{j+1}}\right) T_{j}^{-1}+\left(1-q^{x_{n-1}-x_{n}}\right)\left(1-q^{x_{n-1}+x_{n}}\right) T_{n-1}^{-1} \\
& +w_{+}\left(x_{n}\right)\left(1-q^{x_{n-1}-x_{n}}\right) T_{n}+w_{-}\left(x_{n}\right)\left(1-q^{x_{n-1}+x_{n}}\right) T_{n}^{-1}+U\left(x_{n-1}, x_{n}\right),
\end{aligned}
$$

where

$$
\begin{aligned}
w_{+}(x) & :=\frac{\prod_{0 \leq r \leq 3}\left(1-t_{r} q^{x}\right)}{\left(1-q^{2 x}\right)\left(1-q^{2 x+1}\right)}, \quad w_{-}(x):=\frac{\prod_{0 \leq r \leq 3}\left(1-t_{r}^{-1} q^{x}\right)}{\left(1-q^{2 x}\right)\left(1-q^{2 x-1}\right)}, \\
U(x, y) & :=\sum_{\epsilon \in\{1,-1\}} \frac{c_{\epsilon}\left(1-\epsilon q^{x+1 / 2}\right)}{\left(1-\epsilon q^{y-1 / 2}\right)\left(1-\epsilon q^{-y-1 / 2}\right)},
\end{aligned}
$$

with

$$
c_{\epsilon}:=\frac{1}{2 \sqrt{q^{-1} t_{0} t_{1} t_{2} t_{3}}} \prod_{0 \leq r \leq 3}\left(1-\epsilon q^{-1 / 2} t_{r}\right),
$$

and $T_{j}(j=1, \ldots, n)$ acts on functions $f: \mathbb{R}^{n} \rightarrow \mathbb{C}$ by a unit translation of the $j$ th position variable

$$
\left(T_{j} f\right)\left(x_{1}, \ldots, x_{n}\right)=f\left(x_{1}, \ldots, x_{j-1}, x_{j}+1, x_{j+1}, \ldots, x_{n}\right) .
$$

Here $q$ denotes a scale parameter and the parameters $t_{r}(r=0, \ldots, 3)$ play the role of coupling parameters for the boundary interaction of Askey-Wilson type. Upon setting $t_{2}=-t_{3}=q^{1 / 2}$, the additive potential term $U\left(x_{n-1}, x_{n}\right)$ in $H$ (2.1a)(2.1d) vanishes. The above Toda chain amounts in this case to a difference analog of the previously studied $D_{n}$-type quantum Toda chain with Pöschl-Teller boundary potential I, KJC, O, GLO2]. If we additionally set $t_{0}=-t_{1}=1$, then $w_{+}(x)=$ $w_{-}(x)=1$ and we formally recover a $D_{n}$-type analog of Ruijsenaars' difference 
Toda chain $[\mathrm{KT}, \mathrm{E}, \mathrm{S}, \mathrm{C}]$ that was introduced at the level of classical mechanics by Suris Su1.

\section{Deformed hyperoctahedral $q$-Whittaker functions}

Let $\Lambda$ denote the cone of integer partitions $\lambda=\left(\lambda_{1}, \ldots, \lambda_{n}\right)$ with decreasingly ordered parts $\lambda_{1} \geq \cdots \geq \lambda_{n} \geq 0$, and let $W$ be the hyperoctahedral group formed by the semi-direct product of the symmetric group $S_{n}$ and the $n$-fold product of the cyclic group $\mathbb{Z}_{2} \cong\{1,-1\}$. Elements $w=(\sigma, \epsilon) \in W$ act naturally on $\xi=\left(\xi_{1}, \ldots \xi_{n}\right) \in \mathbb{R}^{n}$ via $w \xi:=\left(\epsilon_{1} \xi_{\sigma_{1}}, \ldots, \epsilon_{n} \xi_{\sigma_{n}}\right)$ (with $\sigma \in S_{n}$ and $\epsilon_{j} \in\{1,-1\}$ for $j=1, \ldots, n)$. A standard basis for the algebra of $W$-invariant trigonometric polynomials on the torus $\mathbb{T}=\mathbb{R}^{n} /\left(2 \pi \mathbb{Z}^{n}\right)$ is given by the hyperoctahedral monomial symmetric functions

$$
m_{\lambda}(\xi):=\sum_{\mu \in W \lambda} e^{i\langle\mu, \xi\rangle}, \quad \lambda \in \Lambda,
$$

where the summation is meant over the orbit of $\lambda$ with respect to the action of $W$ and the bracket $\langle\cdot, \cdot\rangle$ refers to the usual inner product on $\mathbb{R}^{n}$ (so $\langle\mu, \xi\rangle=$ $\left.\mu_{1} \xi_{1}+\cdots+\mu_{n} \xi_{n}\right)$. This monomial basis inherits a natural partial order from the hyperoctahedral dominance ordering of the partitions:

$$
\forall \mu, \lambda \in \Lambda: \quad \mu \leq \lambda \text { iff } \sum_{1 \leq j \leq k} \mu_{j} \leq \sum_{1 \leq j \leq k} \lambda_{j} \text { for } k=1, \ldots, n .
$$

By definition, the basis of deformed hyperoctahedral $q$-Whittaker functions $p_{\lambda}(\xi)$, $\lambda \in \Lambda$ is given by the polynomials of the form

$$
p_{\lambda}(\xi)=m_{\lambda}(\xi)+\sum_{\substack{\mu \in \Lambda \\ \text { with } \mu<\lambda}} c_{\lambda, \mu} m_{\mu}(\xi) \quad\left(c_{\lambda, \mu} \in \mathbb{C}\right)
$$

such that

$$
\left\langle p_{\lambda}, m_{\mu}\right\rangle_{\hat{\Delta}}=0 \quad \text { if } \mu<\lambda
$$

where the inner product

$$
\langle\hat{f}, \hat{g}\rangle_{\hat{\Delta}}:=\int_{\mathbb{A}} \hat{f}(\xi) \overline{\hat{g}(\xi)} \hat{\Delta}(\xi) \mathrm{d} \xi \quad\left(\hat{f}, \hat{g} \in L^{2}(\mathbb{A}, \hat{\Delta}(\xi) \mathrm{d} \xi)\right)
$$

is determined by the weight function

$$
\hat{\Delta}(\xi):=\frac{1}{(2 \pi)^{n}} \prod_{1 \leq j<k \leq n}\left|\left(e^{i\left(\xi_{j}+\xi_{k}\right)}, e^{i\left(\xi_{j}-\xi_{k}\right)}\right)_{\infty}\right|^{2} \prod_{1 \leq j \leq n}\left|\frac{\left(e^{2 i \xi_{j}}\right)_{\infty}}{\prod_{0 \leq r \leq 3}\left(\hat{t}_{r} e^{i \xi_{j}}\right)_{\infty}}\right|^{2}
$$

supported on the hyperoctahedral Weyl alcove

$$
\mathbb{A}:=\left\{\left(\xi_{1}, \xi_{2}, \ldots, \xi_{n}\right) \in \mathbb{R}^{n} \mid \pi>\xi_{1}>\xi_{2}>\cdots>\xi_{n}>0\right\} .
$$

Here $(x)_{m}:=\prod_{l=0}^{m}\left(1-x q^{l}\right)$ and $\left(x_{1}, \ldots, x_{l}\right)_{m}:=\left(x_{1}\right)_{m} \cdots\left(x_{l}\right)_{m}$ refer to standard notations for the $q$-Pochhammer symbols, and it is assumed that

$$
q \in(0,1) \quad \text { and } \quad \hat{t}_{r} \in(-1,1) \backslash\{0\} \quad(r=0, \ldots, 3) .
$$

These deformed hyperoctahedral $q$-Whittaker functions $p_{\lambda}(\xi), \lambda \in \Lambda$ amount to a $t \rightarrow 0$ degeneration of the more general Macdonald-Koorwinder multivariate AskeyWilson polynomials introduced in Ref. $\underline{\mathrm{K}}$ (cf. Appendix $\mathrm{A}$ below). 


\section{Diagonalization}

It is known that the eigenfunctions of Ruijsenaars' open difference Toda chain consist of $A_{n-1}$-type $q$-Whittaker functions given by a $t \rightarrow 0$ limit of the Macdonald symmetric functions GLO1. In this section our aim is to show that an analogous result holds for the chain with Askey-Wilson type boundary interactions from Section 2. upon employing the deformed hyperoctahedral $q$-Whittaker functions from Section 3. To this end it is convenient to reparametrize the boundary parameters of the Toda chain in terms of the $q$-Whittaker deformation parameters (3.6) via

$$
t_{0}=\sqrt{q^{-1} \hat{t}_{0} \hat{t}_{1} \hat{t}_{2} \hat{t}_{3}}, \quad t_{r}=\hat{t}_{r} \hat{t}_{0} / t_{0} \quad(r=1,2,3),
$$

assuming (from now onwards) the additional positivity constraints

$$
\hat{t}_{0}>0 \text { and } \hat{t}_{0} \hat{t}_{1} \hat{t}_{2} \hat{t}_{3}>0 \text {. }
$$

Let $\rho_{0}+\Lambda:=\left\{\rho_{0}+\lambda \mid \lambda \in \Lambda\right\}$ with

$$
\rho_{0}:=\left(\log _{q}\left(t_{0}\right), \ldots, \log _{q}\left(t_{0}\right)\right) \in \mathbb{R}^{n} .
$$

We write $\ell^{2}\left(\rho_{0}+\Lambda, \Delta\right)$ for the Hilbert space of lattice functions $f:\left(\rho_{0}+\Lambda\right) \rightarrow \mathbb{C}$ determined by the inner product

$$
\langle f, g\rangle_{\Delta}:=\sum_{\lambda \in \Lambda} f\left(\rho_{0}+\lambda\right) \overline{g\left(\rho_{0}+\lambda\right)} \Delta_{\lambda} \quad\left(f, g \in \ell^{2}\left(\rho_{0}+\lambda_{n}, \Delta\right)\right),
$$

where

$$
\Delta_{\lambda}:=\frac{\Delta_{0}}{\left(q t_{0}^{2}\right)_{\lambda_{n-1}+\lambda_{n}}}\left(\frac{1-t_{0}^{2} q^{2 \lambda_{n}}}{1-t_{0}^{2}}\right) \prod_{0 \leq r \leq 3} \frac{\left(t_{0} t_{r}\right)_{\lambda_{n}}}{\left(q t_{0} t_{r}^{-1}\right)_{\lambda_{n}}} \prod_{1 \leq j<n} \frac{1}{(q)_{\lambda_{j}-\lambda_{j+1}}}
$$

and

$$
\Delta_{0}:=(q)_{\infty} \prod_{0 \leq r<s \leq 3}\left(\hat{t}_{r} \hat{t}_{s}\right)_{\infty}=(q)_{\infty} \prod_{1 \leq r \leq 3}\left(t_{0} t_{r}, q t_{0} t_{r}^{-1}\right)_{\infty} .
$$

From the limiting behavior for $t \rightarrow 0$ of the orthogonality relations satisfied by the normalized Macdonald-Koornwinder polynomials (A.2a - A.2C), it is immediate that the wave function

$$
\psi_{\xi}\left(\rho_{0}+\lambda\right):=\frac{\left(t_{0}^{2}\right)_{2 \lambda_{n}}}{\prod_{0 \leq r \leq 3}\left(t_{0} t_{r}\right)_{\lambda_{n}}} p_{\lambda}(\xi) \quad(\lambda \in \Lambda, \xi \in \mathbb{A})
$$

satisfies the following orthogonality with respect to the spectral variable $\xi$ :

$$
\int_{\mathbb{A}} \psi\left(\rho_{0}+\lambda\right) \overline{\psi\left(\rho_{0}+\mu\right)} \hat{\Delta}(\xi) \mathrm{d} \xi= \begin{cases}\Delta_{\lambda}^{-1} & \text { if } \lambda=\mu \\ 0 & \text { otherwise. }\end{cases}
$$

In other words, the corresponding Fourier transform $\boldsymbol{F}: \ell^{2}\left(\rho_{0}+\Lambda, \Delta\right) \rightarrow L^{2}(\mathbb{A}, \hat{\Delta} \mathrm{d} \xi)$ given by

$$
(\boldsymbol{F} f)(\xi):=\left\langle f, \psi_{\xi}\right\rangle_{\Delta}=\sum_{\lambda \in \Lambda} f\left(\rho_{0}+\lambda\right) \overline{\psi_{\xi}\left(\rho_{0}+\lambda\right)} \Delta_{\lambda}
$$

$\left(f \in \ell^{2}\left(\rho_{0}+\Lambda, \Delta\right)\right)$ constitutes a Hilbert space isomorphism with an inversion formula of the form

$$
\left(\boldsymbol{F}^{-1} \hat{f}\right)\left(\rho_{0}+\lambda\right)=\left\langle\hat{f}, \overline{\psi\left(\rho_{0}+\lambda\right)}\right\rangle_{\hat{\Delta}}=\int_{\mathbb{A}} \hat{f}(\xi) \psi_{\xi}\left(\rho_{0}+\lambda\right) \hat{\Delta}(\xi) \mathrm{d} \xi
$$

$\left(\hat{f} \in L^{2}(\mathbb{A}, \hat{\Delta} \mathrm{d} \xi)\right)$. We will refer to $\boldsymbol{F}$ (4.6a), 4.6b) as the deformed hyperoctahedral $q$-Whittaker transform. 
The formal Hamiltonian $H$ (2.1a) - 2.1d) restricts to a well-defined discrete difference operator in the space of complex functions on the lattice $\rho_{0}+\Lambda$. Indeed, when $t_{0} \notin\left\{1, q^{1 / 2}\right\}$ it is manifest that for $x=\left(x_{1}, \ldots, x_{n}\right)$ at these lattice points we stay away from the poles in the coefficients of $H$ stemming from the denominators of $w_{ \pm}\left(x_{n}\right)$ and $U\left(x_{n-1}, x_{n}\right)$ and, moreover, that for any $f: \mathbb{R}^{n} \rightarrow \mathbb{C}$ and any $\lambda \in \Lambda$ the value of $(H f)\left(\rho_{0}+\lambda\right)$ depends only on evaluations of $f$ at points of $\rho_{0}+\Lambda$ (due to the vanishing of $\left(1-q^{\lambda_{j}-\lambda_{j+1}}\right)$ at $\lambda_{j}=\lambda_{j+1}(1 \leq j<n)$ and the vanishing of $w_{-}\left(\log _{q}\left(t_{0}\right)+\lambda_{n}\right)$ at $\left.\lambda_{n}=0\right)$ :

$$
(H f)\left(\rho_{0}+\lambda\right)=
$$

where

$$
\begin{aligned}
v_{j}^{+}(\lambda)= & \left(1-q^{\lambda_{j-1}-\lambda_{j}}\right)\left(\frac{\prod_{0 \leq r \leq 3}\left(1-t_{r} t_{0} q^{\lambda_{n}}\right)}{\left(1-t_{0}^{2} q^{2 \lambda_{n}}\right)\left(1-t_{0}^{2} q^{2 \lambda_{n}+1}\right)}\right)^{\delta_{n-j}}, \\
v_{j}^{-}(\lambda)= & \left(1-q^{\lambda_{j}-\lambda_{j+1}}\right)\left(1-t_{0}^{2} q^{\lambda_{n-1}+\lambda_{n}}\right)^{\delta_{n-j}+\delta_{n-1-j}} \\
& \times\left(\frac{\prod_{0 \leq r \leq 3}\left(1-t_{r}^{-1} t_{0} q^{\lambda_{n}}\right)}{\left(1-t_{0}^{2} q^{2 \lambda_{n}}\right)\left(1-t_{0}^{2} q^{2 \lambda_{n}-1}\right)}\right)^{\delta_{n-j}}, \\
u(\lambda)= & \sum_{\epsilon \in\{1,-1\}} \frac{c_{\epsilon}\left(1-\epsilon t_{0} q^{\lambda_{n-1}+1 / 2}\right)}{\left(1-\epsilon t_{0} q^{\lambda_{n}-1 / 2}\right)\left(1-\epsilon t_{0}^{-1} q^{-\lambda_{n}-1 / 2}\right)}
\end{aligned}
$$

with $c_{\epsilon}$ taken from (2.1d). Here $\delta_{k}:=1$ if $k=0$ and $\delta_{k}:=0$ otherwise, the vectors $e_{1}, \ldots, e_{n}$ denote the standard unit basis of $\mathbb{R}^{n}$, and $\lambda_{0}:=+\infty, \lambda_{n+1}:=-\infty$ by convention (so $\left.\left(1-q^{\lambda_{0}-\lambda_{1}}\right)=\left(1-q^{\lambda_{n}-\lambda_{n+1}}\right) \equiv 1\right)$. The action of $H$ on lattice functions in Eq. (4.7) extends continuously from $t_{0} \notin\left\{1, q^{1 / 2}\right\}$ to the full parameter domain determined by Eqs. (4.1), (4.2) and (3.6).

Our main result implements the Hamiltonian under consideration as a selfadjoint operator in the Hilbert space $\ell^{2}\left(\rho_{0}+\Lambda, \Delta\right)$ and provides its spectral decomposition with the aid of the deformed hyperoctahedral $q$-Whittaker transform.

Theorem 1 (Diagonalization). (i). For boundary parameters $t_{r}$ (4.1) determined by the $q$-Whittaker deformation parameters $\hat{t}_{r}$ (3.6), (4.2), the action of the difference Toda Hamiltonian $H$ (2.1a) -2.1d) given by Eq. (4.7) constitutes a bounded self-adjoint operator in the Hilbert space $\ell^{2}\left(\rho_{0}+\Lambda, \Delta\right)$ with purely absolutely continuous spectrum. (ii). The operator in question is diagonalized by the deformed hyperoctahedral q-Whittaker transform $\boldsymbol{F}$ (4.6a), (4.6b):

$$
H=\boldsymbol{F}^{-1} \circ \hat{E} \circ \boldsymbol{F}
$$

where $\hat{E}$ denotes the bounded real multiplication operator acting on $\hat{f} \in L^{2}(\mathbb{A}, \hat{\Delta} d \xi)$ via

$$
(\hat{E} \hat{f})(\xi):=\hat{E}(\xi) \hat{f}(\xi) \quad \text { with } \quad \hat{E}(\xi):=2 \sum_{1 \leq j \leq n} \cos \left(\xi_{j}\right) .
$$

Proof. The first part of the theorem is immediate form the second part. To prove the second part it suffices to verify that the deformed hyperoctahedral $q$-Whittaker 
kernel $\psi_{\xi}$ satisfies the eigenvalue equation $H \psi_{\xi}=\hat{E}(\xi) \psi_{\xi}$, or more explicitly that:

$$
\begin{array}{r}
\sum_{\substack{1 \leq j \leq n \\
\lambda+e_{j} \in \Lambda}} v_{j}^{+}(\lambda) \psi_{\xi}\left(\rho_{0}+\lambda+e_{j}\right)+\sum_{\substack{1 \leq j \leq n \\
\lambda-e_{j} \in \Lambda}} v_{j}^{-}(\lambda) \psi_{\xi}\left(\rho_{0}+\lambda-e_{j}\right) \\
+u(\lambda) \psi_{\xi}\left(\rho_{0}+\lambda\right)=\hat{E}(\xi) \psi_{\xi}\left(\rho_{0}+\lambda\right) .
\end{array}
$$

This eigenvalue equation follows from the Pieri formula for the Macdonald-Koornwinder polynomials (A.4) in the limit $t \rightarrow 0$. Indeed, it is clear that in the Pieri formula $\lim _{t \rightarrow 0} \mathbf{P}_{\lambda}(\xi)=\psi_{\lambda}\left(\rho_{0}+\lambda\right), \lim _{t \rightarrow 0} \hat{\tau}_{j} V_{j}^{+}(\lambda)=v_{j}^{+}(\lambda), \lim _{t \rightarrow 0} \hat{\tau}_{j}^{-1} V_{j}^{-}(\lambda)=v_{j}^{-}(\lambda)$, and one also has that

$$
\lim _{t \rightarrow 0}\left(\sum_{j=1}^{n}\left(\hat{\tau}_{j}+\hat{\tau}_{j}^{-1}\right)-\sum_{\substack{1 \leq j \leq n \\ \lambda+e_{j} \in \Lambda}} V_{j}^{+}(\lambda)-\sum_{\substack{1 \leq j \leq n \\ \lambda-e_{j} \in \Lambda}} V_{j}^{-}(\lambda)\right)=u(\lambda) .
$$

This last limit formula is not evident but can be deduced from the following rational identity in $q^{x_{1}}, \ldots, q^{x_{n}}$ :

$$
\begin{aligned}
& \sum_{j=1}^{n}\left(\hat{\tau}_{j}^{-1}-\hat{\tau}_{1}^{-1} w_{+}\left(x_{j}\right) \prod_{\substack{1 \leq k \leq n \\
k \neq j}} \frac{1-t q^{x_{j}+x_{k}}}{1-q^{x_{j}+x_{k}}} \frac{1-t q^{x_{j}-x_{k}}}{1-q^{x_{j}-x_{k}}}\right)+ \\
& \sum_{j=1}^{n}\left(\hat{\tau}_{j}-\hat{\tau}_{1} w_{-}\left(x_{j}\right) \prod_{\substack{1 \leq k \leq n \\
k \neq j}} \frac{1-t^{-1} q^{x_{j}+x_{k}}}{1-q^{x_{j}+x_{k}}} \frac{1-t^{-1} q^{x_{j}-x_{k}}}{1-q^{x_{j}-x_{k}}}\right) \\
& =C_{t} \sum_{\epsilon \in\{1,-1\}} \prod_{0 \leq r \leq 3}\left(1-\epsilon t_{r} q^{-1 / 2}\right)\left(1-\prod_{j=1}^{n} \frac{1-\epsilon t q^{x_{j}-1 / 2}}{1-\epsilon q^{x_{j}-1 / 2}} \frac{1-\epsilon t^{-1} q^{x_{j}+1 / 2}}{1-\epsilon q^{x_{j}+1 / 2}}\right),
\end{aligned}
$$

where $C_{t}=-\frac{1}{2} t \hat{t}_{0}^{-1}(1-t)^{-1}\left(1-q^{-1} t\right)^{-1}$, upon replacing $q^{x_{j}}$ by $\tau_{j} q^{\lambda_{j}}(j=1, \ldots, n)$ and performing the limit $t \rightarrow 0$. To infer the rational identity itself, one exploits the hyperoctahedral symmetry in the variables $x_{1}, \ldots x_{n}$ and checks that - as a function of $x_{j}$ (with the remaining variables fixed in a generic configuration) - the residues at the (simple) poles on both sides coincide. Hence, the difference of both rational expressions amounts to a $W$-invariant Laurent polynomial in $q^{x_{1}}, \ldots, q^{x_{n}}$. The Laurent polynomial in question must actually vanish, as the rational expressions under consideration tend to 0 for $x_{j}=(n+1-j) c$ in the limit $c \rightarrow+\infty$.

\section{INTEGRABILITY}

The quantum integrability of the difference Toda Hamiltonian $H$ (2.1a - 2.1d) is an immediate consequence of the diagonalization in Theorem 1 . In effect, a complete system of commuting quantum integrals in the Hilbert space $\ell^{2}\left(\rho_{0}+\Lambda, \Delta\right)$ is given by the bounded self-adjoint operators

$$
H_{l}:=\boldsymbol{F}^{-1} \circ \hat{E}_{l} \circ \boldsymbol{F}, \quad l=1, \ldots, n,
$$

where $\hat{E}_{l}: L^{2}(\mathbb{A}, \hat{\Delta} \mathrm{d} \xi) \rightarrow L^{2}(\mathbb{A}, \hat{\Delta} \mathrm{d} \xi)$ denotes the real multiplication operator by $\hat{E}_{l}(\xi):=m_{\omega_{l}}(\xi)$ with $\omega_{l}:=e_{1}+\cdots+e_{l}$ (so $H_{1}=H$ ). The operator $H_{l}$ (5.1) acts on $f \in \ell^{2}\left(\rho_{0}+\Lambda, \Delta\right)$ as a difference operator of the form

$$
\left(H_{l} f\right)\left(\rho_{0}+\lambda\right)=\sum_{\substack{J \subset\{1, \ldots, n\}, 0 \leq|J| \leq l \\ \epsilon_{j} \in\{1,-1\}, j \in J ; \lambda+e_{\epsilon J} \in \Lambda}} C_{\epsilon J}^{(l)}(\lambda) f\left(\rho_{0}+\lambda+e_{\epsilon J}\right),
$$


where $e_{\epsilon J}:=\sum_{j \in J} \epsilon_{j} e_{j},|J|$ denotes the cardinality of $J \subset\{1, \ldots, n\}$, and the coefficients

$$
C_{\epsilon J}^{(l)}(\lambda)=\lim _{t \rightarrow 0} C_{\epsilon J, t}^{(l)}(\lambda)
$$

arise as $t \rightarrow 0$ limits of the expansion coefficients in the corresponding Pieri formula for the normalized Macdonald-Koornwinder polynomials $\mathbf{P}_{\lambda}(\xi)$ (A.1a), A.1b) (cf. [D3, Sec. 6]):

$$
\hat{E}_{l}(\xi) \mathbf{P}_{\lambda}(\xi)=\sum_{\substack{J \subset\{1, \ldots, n\}, 0 \leq|J| \leq l \\ \epsilon_{j} \in\{1,-1\}, j \in J ; \lambda+e_{\epsilon} \in \Lambda}} C_{\epsilon J, t}^{(l)}(\lambda) \mathbf{P}_{\lambda+e_{\epsilon}}(\xi) .
$$

Notice in this connection that the Pieri expansion coefficients

$$
C_{\epsilon J, t}^{(l)}(\lambda)=\boldsymbol{\Delta}_{\lambda+e_{\epsilon J}} \int_{\mathbb{A}} \hat{E}_{l}(\xi) \mathbf{P}_{\lambda}(\xi) \overline{\mathbf{P}_{\lambda+e_{\epsilon J}}(\xi)} \hat{\boldsymbol{\Delta}}(\xi) \mathrm{d} \xi
$$

are continuous at $t=0$, because the Macdonald-Koornwinder weight function $\hat{\boldsymbol{\Delta}}(\xi)$ and (thus) the polynomials $\mathbf{P}_{\lambda}(\xi), \lambda \in \Lambda$ are continuous at this parameter value (cf. Appendix A).

In practice it turns out to be very tedious to compute the $t \rightarrow 0$ limiting coefficients $C_{\epsilon J}^{(l)}(\lambda)$ explicitly with the aid of the known explicit Pieri formulas for the Macdonald-Koornwinder polynomials in [D3, Sec. 6] beyond $l=1$. For a particular second quantum integral belonging to the commutative algebra generated by $H_{1}, \ldots, H_{n}$, however, the required computation results to be surprisingly straightforward. More specifically: from the $t \rightarrow 0$ limiting behavior of the $r=n$ (top) Pieri formula for the Macdonald-Koornwinder polynomials in Theorem 6.1 of [D3], one readily deduces that the action on $f \in \ell^{2}\left(\rho_{0}+\Lambda, \Delta\right)$ of the operator $H_{Q}:=\boldsymbol{F}^{-1} \circ \hat{Q} \circ \boldsymbol{F}$, where $\hat{Q}$ refers to the self-adjoint multiplication operator in $L^{2}(\mathbb{A}, \hat{\Delta} \mathrm{d} \xi)$ by

$$
\hat{Q}(\xi):=\prod_{j=1}^{n}\left(2 \cos \left(\xi_{j}\right)-\hat{t}_{0}-\hat{t}_{0}^{-1}\right)
$$

is given explicitly by

$$
\sum_{\substack{J_{+} \cup J_{-} \cup K_{+} \cup K_{-}=\{1, \ldots, n\} \\\left|J_{+}\right|+\left|J_{-}\right|+\left|K_{+}\right|+\left|K_{-}\right|=n \\ \lambda+e_{J_{+}}-e_{J_{-}} \in \Lambda}}^{\left(H_{Q} f\right)\left(\rho_{0}+\lambda\right)=} u_{K_{+}, K_{-}}(\lambda) v_{J_{+}, J_{-}}(\lambda) f\left(\rho_{0}+\lambda+e_{J_{+}}-e_{J_{-}}\right)
$$

with

$$
\begin{aligned}
& v_{J_{+}, J_{-}}(\lambda)=\prod_{\substack{j \in J_{+} \\
j-1 \notin J_{+}}}\left(1-q^{\lambda_{j-1}-\lambda_{j}}\right) \prod_{\substack{j \in J_{-} \\
j+1 \notin J_{-}}}\left(1-q^{\lambda_{j}-\lambda_{j+1}-\delta_{J_{+}}(j+1)}\right) \\
& \times\left(1-t_{0}^{2} q^{\lambda_{n-1}+\lambda_{n}}\right)^{\delta_{J_{+}^{c}}(n-1) \delta_{J_{+}^{c}}(n)-\delta_{J_{+}^{c}}^{J_{+}} \cap J_{-}^{c}(n-1) \delta_{J_{+}^{c} \cap J_{-}^{c}}(n)} \\
& \times\left(1-t_{0}^{2} q^{\lambda_{n-1}+\lambda_{n}-1}\right)^{\delta_{J_{-}}(n-1) \delta_{J_{-}}(n)} w_{+}\left(\lambda_{n}\right)^{\delta_{J_{+}}(n)} w_{-}\left(\lambda_{n}\right)^{\delta_{J_{-}}(n)}
\end{aligned}
$$


and

$$
\begin{aligned}
u_{K_{+}, K_{-}}(\lambda) & =\left(-\hat{t}_{0}\right)^{\left|K_{-}\right|-\left|K_{+}\right|} \prod_{\substack{k \in K_{+} \\
k-1 \in K_{-}}}\left(1-q^{\lambda_{k-1}-\lambda_{k}}\right) \prod_{\substack{k \in K_{+} \\
k+1 \in K_{-}}}\left(1-q^{\lambda_{k}-\lambda_{k+1}+1}\right) \\
& \times\left(1-t_{0}^{2} q^{\lambda_{n-1}+\lambda_{n}+1}\right)^{\delta_{K_{+}}(n-1) \delta_{K_{+}}(n)}\left(1-t_{0}^{2} q^{\lambda_{n-1}+\lambda_{n}}\right)^{\delta_{K_{-}}(n-1) \delta_{K_{-}}(n)} \\
& \times w_{+}\left(\lambda_{n}\right)^{\delta_{K_{+}}(n)} w_{-}\left(\lambda_{n}\right)^{\delta_{K_{-}}(n)} .
\end{aligned}
$$

Here $\delta_{J}:\{1, \ldots, n\} \rightarrow\{0,1\}$ denotes the characteristic function of $J \subset\{1, \ldots, n\}$ and $J^{c}=\{1, \ldots, n\} \backslash J$.

Corollary 2. The difference Toda Hamiltonians $H$ (4.7) and $H_{Q}$ (5.3) are bounded, self-adjoint, commuting operators in $\ell^{2}\left(\rho_{0}+\Lambda, \Delta\right)$ for which the deformed hyperoctahedral $q$-Whittaker functions $\psi_{\xi}$ (4.4) constitute a complete system of (generalized) joint eigenfunctions corresponding to the eigenvalues $\hat{E}(\xi)$ and $\hat{Q}(\xi)$, respectively.

\section{Bispectral DUAL System}

For $t \rightarrow 0$ the Macdonald-Koornwinder $q$-difference equation (A.3) amounts to the following eigenvalue equation satisfied by the deformed hyperoctahedral $q$ Whittaker functions:

$$
\hat{H} p_{\lambda}=\left(q^{-\lambda_{1}}-1\right) p_{\lambda} \quad(\lambda \in \Lambda)
$$

with

$$
\left.\hat{H}=\sum_{j=1}^{n}\left(\hat{v}_{j}(\xi)\left(\hat{T}_{j, q}-1\right)+\hat{v}_{j}(-\xi) \hat{T}_{j, q}^{-1}-1\right)\right)
$$

and

$$
\hat{v}_{j}(\xi)=\frac{\prod_{0 \leq r \leq 3}\left(1-\hat{t}_{r} e^{i \xi_{j}}\right)}{\left(1-e^{2 i \xi_{j}}\right)\left(1-q e^{2 i \xi_{j}}\right)} \prod_{\substack{1 \leq k \leq n \\ k \neq j}}\left(1-e^{i\left(\xi_{j}+\xi_{k}\right)}\right)^{-1}\left(1-e^{i\left(\xi_{j}-\xi_{k}\right)}\right)^{-1},
$$

where $\hat{T}_{j, q}$ acts on trigonometric (Laurent) polynomials $\hat{p}\left(e^{i \xi_{1}}, \ldots, e^{i \xi_{n}}\right)$ by a $q$-shift of the $j$ th variable:

$$
\left(\hat{T}_{j, q} \hat{p}\right)\left(e^{i \xi_{1}}, \ldots, e^{i \xi_{n}}\right):=\hat{p}\left(e^{i \xi_{1}}, \ldots, e^{i \xi_{j-1}}, q e^{i \xi_{j}}, e^{i \xi_{j+1}}, \ldots, e^{i \xi_{n}}\right) .
$$

The following proposition is now immediate.

Proposition 3 (Bispectral Dual Hamiltonian). The $t=0$ Macdonald-Koornwinder $q$-difference operator $\hat{H}$ (6.2a), (6.2b) constitutes a nonnegative unbounded self-adjoint operator with purely discrete spectrum in $L^{2}(\mathbb{A}, \hat{\Delta} d \xi)$ that is diagonalized by the (inverse) deformed hyperoctahedral $q$-Whittaker transform $\boldsymbol{F}$ (4.6a), 4.6b):

$$
\hat{H}=\boldsymbol{F} \circ E \circ \boldsymbol{F}^{-1},
$$

where $E$ denotes the self-adjoint multiplication operator in $\ell^{2}\left(\rho_{0}+\Lambda, \Delta\right)$ of the form

$$
(E f)\left(\rho_{0}+\lambda\right):=\left(q^{-\lambda_{1}}-1\right) f\left(\rho_{0}+\lambda\right) \quad(\lambda \in \Lambda)
$$

(for $f \in \ell^{2}\left(\rho_{0}+\Lambda, \Delta\right)$ with $\left.\langle E f, E f\rangle_{\Delta}<\infty\right)$. 
One learns from Theorem 1 and Proposition 3 that the eigenfunction transforms diagonalizing the difference Toda Hamiltonian $H$ (4.7) and the $t=0$ MacdonaldKoornwinder difference operator $\hat{H}$ (6.2a), 6.2b are inverses of each other. This fact encodes the bispectral duality of the operators under consideration in the sense of Duistermaat and Grünbaum [DG, G]: the kernel function $\psi_{\xi}\left(\rho_{0}+\lambda\right)$ of the deformed hyperoctahedral $q$-Whittaker transform $\boldsymbol{F}$ (4.6a), (4.6b) simultaneously solves the corresponding eigenvalue equations for $H$ and $\hat{H}$ in the discrete variable $\rho_{0}+\lambda$ and the spectral variable $\xi$, respectively.

Explicit commuting quantum integrals for the dual Hamiltonian $\hat{H}$ (6.2a, $6.2 \mathrm{~b}$ ) are obtained as a $t \rightarrow 0$ degeneration of the commuting difference operators in D3. Thm. 5.1]:

$$
\hat{H}_{l}=\sum_{\substack{J \subset\{1, \ldots, n\}, 0 \leq|J| \leq l \\ \epsilon_{j} \in\{1,-1\}, j \in J}} \hat{U}_{J^{c}, l-|J|} \hat{V}_{\epsilon J} \hat{T}_{\epsilon J, q}, \quad l=1, \ldots, n
$$

with $\hat{T}_{\epsilon J, q}:=\prod_{j \in J} \hat{T}_{j, q}^{\epsilon_{j}}$ and

$$
\begin{aligned}
& \hat{V}_{\epsilon J}:= \prod_{j \in J} \frac{\prod_{0 \leq r \leq 3}\left(1-\hat{t}_{r} e^{i \epsilon_{j} \xi_{j}}\right)}{\left(1-e^{2 i \epsilon_{j} \xi_{j}}\right)\left(1-q e^{\left.2 i \epsilon_{j} \xi_{j}\right)}\right.} \prod_{\substack{j \in J \\
k \notin J}}\left(1-e^{i\left(\epsilon_{j} \xi_{j}+\xi_{k}\right)}\right)^{-1}\left(1-e^{i\left(\epsilon_{j} \xi_{j}-\xi_{k}\right)}\right)^{-1} \\
& \times \prod_{\substack{j, k \in J \\
j<k}}\left(1-e^{i\left(\epsilon_{j} \xi_{j}+\epsilon_{k} \xi_{k}\right)}\right)^{-1}\left(1-q e^{i\left(\epsilon_{j} \xi_{j}+\epsilon_{k} \xi_{k}\right)}\right)^{-1}, \\
& \hat{U}_{K, p}:=(-1)^{p} \sum_{\substack{I \subset K,|I|=p \\
\epsilon_{j} \in\{1,-1\}, j \in I}}\left(\prod_{j \in I} \frac{\prod_{0 \leq r \leq 3}\left(1-\hat{t}_{r} e^{i \epsilon_{j} \xi_{j}}\right)}{\left(1-e^{2 i \epsilon_{j} \xi_{j}}\right)\left(1-q e^{\left.2 i \epsilon_{j} \xi_{j}\right)}\right.}\right. \\
& \times \prod_{\substack{j \in I \\
k \in K \backslash I}}\left(1-e^{i\left(\epsilon_{j} \xi_{j}+\xi_{k}\right)}\right)^{-1}\left(1-e^{i\left(\epsilon_{j} \xi_{j}-\xi_{k}\right)}\right)^{-1} \\
&\left.\times \prod_{\substack{j, k \in I \\
j<k}}\left(1-e^{i\left(\epsilon_{j} \xi_{j}+\epsilon_{k} \xi_{k}\right)}\right)^{-1}\left(1-q^{-1} e^{-i\left(\epsilon_{j} \xi_{j}+\epsilon_{k} \xi_{k}\right)}\right)^{-1}\right)
\end{aligned}
$$

(so $\hat{H}_{1}=\hat{H}$ ). The diagonalization in Proposition 3 now generalizes to the complete system of commuting quantum integrals $\hat{H}_{1}, \ldots, \hat{H}_{n}$ as follows.

Theorem 4 (Bispectral Dual System). Let $E_{l}(1 \leq l \leq n)$ denote the self-adjoint multiplication operator in $\ell^{2}\left(\rho_{0}+\Lambda, \Delta\right)$ given by

$$
\left(E_{l} f\right)\left(\rho_{0}+\lambda\right):=E_{\lambda, l} f\left(\rho_{0}+\lambda\right) \quad(\lambda \in \Lambda)
$$

(on the domain of $f \in \ell^{2}\left(\rho_{0}+\Lambda, \Delta\right)$ for which $\left.\left\langle E_{l} f, E_{l} f\right\rangle_{\Delta}<\infty\right)$, where

$$
E_{\lambda, l}:=q^{-\lambda_{1}-\lambda_{2} \cdots-\lambda_{l-1}}\left(q^{-\lambda_{l}}-1\right)+t_{0}^{2} q^{-\lambda_{1}-\lambda_{2} \cdots-\lambda_{n-1}}\left(q^{\lambda_{n}}-1\right) \delta_{n-l} .
$$

The q-difference operators $\hat{H}_{l}$ (6.4) constitute nonnegative unbounded self-adjoint operators with purely discrete spectra in $L^{2}(\mathbb{A}, \hat{\Delta} d \xi)$ that are simultaneously diagonalized by the (inverse) deformed hyperoctahedral q-Whittaker transform $\boldsymbol{F}$ (4.6a), (4.6b) :

$$
\hat{H}_{l}=\boldsymbol{F} \circ E_{l} \circ \boldsymbol{F}^{-1}, \quad l=1, \ldots, n .
$$


Proof. It suffices to verify that

$$
\hat{H}_{l} p_{\lambda}=E_{\lambda, l} p_{\lambda} \quad(\lambda \in \Lambda, l=1, \ldots, n) .
$$

This is achieved by multiplying the $l$ th eigenvalue equation in Eq. (5.8) of [D3] by a scaling factor $t^{l(n-l)+l(l-1) / 2}$ and performing the limit $t \rightarrow 0$. Indeed, since the Macdonald-Koornwinder polynomial $\mathbf{p}_{\lambda}$ converges to the deformed hyperoctahedral $q$-Whittaker function $p_{\lambda}$, we see from the explicit formulas for the operators in question that the LHS of the cited eigenvalue equation converges in this limit manifestly to $\hat{H}_{l} p_{\lambda}$ (up to an overall factor $t_{0}^{l}$ ). Hence, the RHS must also have a finite limit for $t \rightarrow 0$, which confirms that $p_{\lambda}$ is an eigenfunction of $\hat{H}_{l}$ (using again that $\mathbf{p}_{\lambda} \stackrel{t \rightarrow 0}{\longrightarrow} p_{\lambda}$ ). For $l>1$ it is not obvious from [D3, Eq. (5.5)] that the (limiting) eigenvalue is indeed given by $E_{\lambda, l}(\underline{6.5 \mathrm{~b}})$, but this can be deduced quite easily from the asymptotics of $m_{\lambda}$ and $\hat{H}_{l} m_{\lambda}$ at $\xi=-c i \rho, \rho:=(n, n-1, \ldots, 2,1)$ for $c \rightarrow+\infty$. Indeed, one readily computes that for $c \rightarrow+\infty: m_{\lambda}=e^{\langle\lambda, \rho\rangle c}(1+o(1))$ and $\hat{H}_{l} m_{\lambda}=E_{\lambda, l} e^{\langle\lambda, \rho\rangle c}(1+o(1))$ (using the explicit formula for $\hat{H}_{l}$ and the asymptotics

$$
\frac{\prod_{0 \leq r \leq 3}\left(1-\hat{t}_{r} e^{i \epsilon \xi_{j}}\right)}{\left(1-e^{2 i \epsilon \xi_{j}}\right)\left(1-q e^{2 i \epsilon \xi_{j}}\right)} \stackrel{c \rightarrow+\infty}{\longrightarrow}\left\{\begin{array}{ll}
t_{0}^{2} & \text { if } \epsilon=1 \\
1 & \text { if } \epsilon=-1
\end{array} \quad(1 \leq j \leq n)\right.
$$

and

$$
\left(1-q^{a} e^{i \epsilon\left(\xi_{j} \pm \xi_{k}\right)}\right)^{-1} \stackrel{c \rightarrow+\infty}{\longrightarrow}\left\{\begin{array}{ll}
0 & \text { if } \epsilon=1 \\
1 & \text { if } \epsilon=-1
\end{array} \quad(1 \leq j<k \leq n),\right.
$$

where $a \in\{1,0,-1\})$. But then also $p_{\lambda}=e^{\langle\lambda, \rho\rangle c}(1+o(1))$ and $\hat{H}_{l} p_{\lambda}=E_{\lambda, l} e^{\langle\lambda, \rho\rangle c}(1+$ $o(1))$ for $c \rightarrow+\infty$ by the triangularity (3.3a) and the property that $\langle\mu, \rho\rangle\langle\langle\lambda, \rho\rangle$ if $\mu<\lambda$. The upshot is that the eigenvalue of $\hat{H}_{l}$ on the eigenpolynomial $p_{\lambda}$ must be equal to $E_{\lambda, l}$.

The $q$-difference operators $\hat{H}_{l}$ (6.4) commute in the space of $W$-invariant trigonometric polynomials on $\mathbb{T}$. It is clear from Theorem 4 that this commutativity extends in the Hilbert space in the resolvent sense: for

$$
z_{l} \notin \sigma\left(\hat{H}_{l}\right):=\left\{E_{\lambda, l} \mid \lambda \in \Lambda\right\} \subset[0,+\infty) \quad(l=1, \ldots, n)
$$

the resolvents $\left(\hat{H}_{1}-z_{1} \mathrm{I}\right)^{-1}, \ldots,\left(\hat{H}_{n}-z_{n} \mathrm{I}\right)^{-1}$ of the unbounded operators $\hat{H}_{1}, \ldots, \hat{H}_{n}$ mutually commute as bounded operators in $L^{2}(\mathbb{A}, \hat{\Delta} \mathrm{d} \xi)$.

Theorem 4 and Section 5 lift the bispectral duality of $H$ (4.7) and $\hat{H}$ (6.2a), (6.2b) to the complete systems of commuting quantum integrals. The bispectral dual integrable system $\hat{H}_{1}, \ldots, \hat{H}_{n}$ associated with our difference Toda chain can actually be identified as the strong-coupling limit $\left(t=q^{g}, g \rightarrow+\infty\right)$ of a trigonometric Ruijsenaars-type difference Calogero-Moser system with hyperoctahedral symmetry [D2]. Analogous bispectral dual systems were linked previously to the open quantum Toda chain and Ruijsenaars' open difference Toda chain. Specifically, the open quantum Toda chain and the strong-coupling limit of Ruijsenaars' rational difference Calogero-Moser system turn out to be bispectral duals of each other $\mathrm{B}, \mathrm{HR}, \mathrm{Sk} 2, \mathrm{Kz}$, and the same holds true for Ruijsenaars' open difference Toda chain and the $t=0$ trigonometric/hyperbolic Ruijsenaars-Macdonald operators GLO1, HR, BC. Dualities of this type were actually first established for the corresponding particle systems within the realms of classical mechanics: the actionangle transforms linearizing the open Toda chain and the strong-coupling limit of 
the rational Ruijsenaars-Schneider system are the inverses of each other and the same holds true for the action-angle transforms for Ruijsenaars' open relativistic Toda chain and the strong-coupling limit of the hyperbolic Ruijsenaars-Schneider system [R1, F].

\section{Parameter Reductions}

As already anticipated at the end of Section 2, for $\hat{t}_{2}=-\hat{t}_{3}=q^{1 / 2}$ and $\hat{t_{0}}=$ $-\hat{t}_{1} \rightarrow 1$ (so $t_{0}=-t_{1} \rightarrow 1$ and $t_{2}=-t_{3} \rightarrow q^{1 / 2}$ ) the difference Toda Hamiltonian $H$ (4.7) and the deformed hyperoctahedral $q$-Whittaker functions $p_{\lambda}(\xi), \lambda \in \Lambda$ degenerate to a difference Toda Hamiltonian and $q$-Whittaker functions of type $D_{n}$ Su1, KT, E, S, C]. Even though formally these limiting values of the parameters do not respect our restriction that $\hat{t}_{r} \in(-1,1) \backslash\{0\}$ (for $r=0, \ldots, 3$ ), it is readily inferred from the formulas that the results of Sections 3 7 nevertheless remain valid at this specialization of the parameters.

In this section we are concerned with the behavior for $\hat{t}_{0} \rightarrow 0$. In this limit, the difference Toda chain turns out to be governed by a Hamiltonian of the form

$$
\begin{aligned}
\mathrm{H}= & T_{1}+\sum_{j=2}^{n}\left(1-q^{x_{j-1}-x_{j}}\right) T_{j}+\sum_{j=1}^{n-1}\left(1-q^{x_{j}-x_{j+1}}\right) T_{j}^{-1} \\
& +\left(\prod_{1 \leq r<s \leq 3}\left(1-\hat{t}_{r} \hat{t}_{s} q^{x_{n}-1}\right)\right)\left(1-q^{x_{n}}\right) T_{n}^{-1} \\
& +\left(\hat{t}_{1}+\hat{t}_{2}+\hat{t}_{3}\right) q^{x_{n}}+\hat{t}_{1} \hat{t}_{2} \hat{t}_{3} q^{2 x_{n}}\left(q^{x_{n-1}-x_{n}}+q^{-x_{n}-1}-1-q^{-1}\right) .
\end{aligned}
$$

When $\hat{t}_{3}=0$, the Hamiltonian in question constitutes a Ruijsenaars-type difference counterpart of the quantum Toda chain with one-sided boundary potentials of Morse type [Sk1, I]. If in addition $\hat{t}_{2}=-1$, then the difference Toda chain under consideration amounts to a quantization of a relativistic Toda chain with boundary potentials introduced by Suris [Su1, [KT. For $\hat{t}_{1}=\hat{t}_{2}=\hat{t}_{3}=0$ and for $\hat{t}_{1}=-\hat{t}_{2}=q^{1 / 2}$ with $\hat{t}_{3}=-1$, we recover in turn hyperoctahedral difference Toda chains of type $B_{n}$ and $C_{n}$ that are diagonalized by $q$-Whittaker functions of type $C_{n}$ and $B_{n}$, respectively $[\mathrm{E}, \mathrm{S}, \mathrm{C}]$. Again, even though formally none of these specializations respect our restriction that $\hat{t}_{r} \in(-1,1) \backslash\{0\}$ (for $r=1,2,3$ ), it is clear that the formulas below in fact do remain valid.

7.1. Deformed hyperoctahedral $q$-Whittaker function. For $\hat{t}_{0} \rightarrow 0$, the deformed hyperoctahedral $q$-Whittaker functions $p_{\lambda}(\xi)$ (3.3a), (3.3b) degenerate into a three-parameter family of orthogonal polynomials $\mathrm{p}_{\lambda}(\xi), \lambda \in \Lambda$ associated with the weight function

$$
\hat{\Delta}(\xi)=\frac{1}{(2 \pi)^{n}} \prod_{1 \leq j<k \leq n}\left|\left(e^{i\left(\xi_{j}+\xi_{k}\right)}, e^{i\left(\xi_{j}-\xi_{k}\right)}\right)_{\infty}\right|^{2} \prod_{1 \leq j \leq n}\left|\frac{\left(e^{2 i \xi_{j}}\right)_{\infty}}{\prod_{1 \leq r \leq 3}\left(\hat{t}_{r} e^{i \xi_{j}}\right)_{\infty}}\right|^{2} .
$$

The orthogonality relations for these polynomials read (cf. Eq. (4.5))

$$
\int_{\mathbb{A}} \mathrm{p}_{\lambda}(\xi) \overline{\mathrm{p}_{\mu}(\xi)} \hat{\Delta}(\xi) \mathrm{d} \xi= \begin{cases}\Delta_{\lambda}^{-1} & \text { if } \lambda=\mu \\ 0 & \text { otherwise }\end{cases}
$$

where

$$
\Delta_{\lambda}=\frac{\Delta_{0}}{(q)_{\lambda_{n}} \prod_{1 \leq r<s \leq 3}\left(\hat{t}_{r} \hat{t}_{s}\right)_{\lambda_{n}}} \prod_{1 \leq j<n} \frac{1}{(q)_{\lambda_{j}-\lambda_{j+1}}}
$$


with

$$
\Delta_{0}=(q)_{\infty} \prod_{1 \leq r<s \leq 3}\left(\hat{t}_{r} \hat{t}_{s}\right)_{\infty}
$$

For $n=1$, the limit $p_{\lambda} \stackrel{\hat{t}_{0} \rightarrow 0}{\longrightarrow} \mathrm{p}_{\lambda}$ amounts to a well-known reduction from the Askey-Wilson polynomials to the continuous dual $q$-Hahn polynomials [KLS].

7.2. Hamiltonian. The difference Toda eigenvalue equation $H \psi_{\xi}=\hat{E}(\xi) \psi_{\xi}$ becomes in the limit $\hat{t}_{0} \rightarrow 0$ of the form $\mathrm{H} \phi_{\xi}=\hat{E}(\xi) \phi_{\xi}$ with $\phi_{\xi}: \Lambda \rightarrow \mathbb{C}$ given by $\phi_{\xi}(\lambda)=\mathrm{p}_{\lambda}(\xi)(\xi \in \mathbb{A}, \lambda \in \Lambda)$, where $\mathrm{H}$ (7.1) acts on $f: \Lambda \rightarrow \mathbb{C}$ via

$$
(\mathrm{H} f)(\lambda)=\sum_{\substack{1 \leq j \leq n \\ \lambda+e_{j} \in \Lambda}} v_{j}^{+}(\lambda) f\left(\lambda+e_{j}\right)+\sum_{\substack{1 \leq j \leq n \\ \lambda-e_{j} \in \Lambda}} v_{j}^{-}(\lambda) f\left(\lambda-e_{j}\right)+u(\lambda) f(\lambda),
$$

with

$$
\begin{aligned}
v_{j}^{+}(\lambda) & =\left(1-q^{\lambda_{j-1}-\lambda_{j}}\right), \\
v_{j}^{-}(\lambda) & =\left(1-q^{\lambda_{j}-\lambda_{j+1}}\right)\left(\left(1-q^{\lambda_{n}}\right) \prod_{1 \leq r<s \leq 3}\left(1-\hat{t}_{r} \hat{t}_{s} q^{\lambda_{n}-1}\right)\right)^{\delta_{n-j}}, \\
u(\lambda) & =\left(\hat{t}_{1}+\hat{t}_{2}+\hat{t}_{3}\right) q^{\lambda_{n}}+\hat{t}_{1} \hat{t}_{2} \hat{t}_{3} q^{2 \lambda_{n}}\left(q^{\lambda_{n-1}-\lambda_{n}}+q^{-\lambda_{n}-1}-1-q^{-1}\right)
\end{aligned}
$$

(subject to the convention that $\lambda_{0}=+\infty$ and $\lambda_{n+1}=-\infty$ ).

7.3. Diagonalization and integrability. Let $\mathbf{F}: \ell^{2}(\Lambda, \Delta) \rightarrow L^{2}(\mathbb{A}, \hat{\Delta} \mathrm{d} \xi)$ denote the $\left(\hat{t}_{0} \rightarrow 0\right.$ degenerate) Hilbert space isomorphism determined by the orthogonal basis $\mathrm{p}_{\lambda}, \lambda \in \Lambda$ :

$$
(\mathbf{F} f)(\xi)=\left\langle f, \phi_{\xi}\right\rangle_{\Delta}=\sum_{\lambda \in \Lambda} f(\lambda) \overline{\phi_{\xi}(\lambda)} \Delta_{\lambda}
$$

$\left(f \in \ell^{2}(\Lambda, \Delta)\right)$ with

$$
\left(\mathbf{F}^{-1} \hat{f}\right)(\lambda)=\langle\hat{f}, \overline{\phi(\lambda)}\rangle_{\hat{\Delta}}=\int_{A} \hat{f}(\xi) \phi_{\xi}(\lambda) \hat{\Delta}(\xi) \mathrm{d} \xi
$$

$\left(\hat{f} \in L^{2}(\mathbb{A}, \hat{\Delta} \mathrm{d} \xi)\right)$, and let $\hat{E}_{l}: L^{2}(\mathbb{A}, \hat{\Delta} \mathrm{d} \xi) \rightarrow L^{2}(\mathbb{A}, \hat{\Delta} \mathrm{d} \xi)(l=1, \ldots, n)$ be the multiplication operators defined in accordance with Section 5 .

The commuting bounded self-adjoint operators $\mathrm{H}_{1}, \ldots, \mathrm{H}_{n}$ (with absolutely continuous spectra) in $\ell^{2}(\Lambda, \Delta)$ given by

$$
\mathrm{H}_{l}=\mathbf{F}^{-1} \circ \hat{E}_{l} \circ \mathbf{F}, \quad l=1, \ldots, n,
$$

constitute a complete system of quantum integrals for the difference Toda Hamiltonian $\mathrm{H}_{1}=\mathrm{H}(7.3)$.

7.4. Bispectral dual system. Let $\hat{\mathrm{H}}_{1}, \ldots, \hat{\mathrm{H}}_{n}$ denote the commuting $q$-difference operators in Eq. (6.4) with $\hat{t}_{0}=0$ and let $\mathrm{E}_{1}, \ldots, \mathrm{E}_{n}$ be the self-adjoint multiplication operators in $\ell^{2}(\Lambda, \Delta)$ given by (cf. Eqs. (6.5a), (6.5b)

$$
\left(\mathrm{E}_{l} f\right)(\lambda)=\mathrm{E}_{\lambda, l} f(\lambda) \quad(\lambda \in \Lambda, l=1, \ldots, n)
$$

(on the domain of $f \in \ell^{2}(\Lambda, \Delta)$ for which $\left\langle\mathrm{E}_{l} f, \mathrm{E}_{l} f\right\rangle_{\Delta}<\infty$ ), with

$$
\mathrm{E}_{\lambda, l}=q^{-\lambda_{1}-\lambda_{2} \cdots-\lambda_{l-1}}\left(q^{-\lambda_{l}}-1\right) .
$$

Then one has that

$$
\hat{\mathrm{H}}_{l}=\mathbf{F} \circ \mathrm{E}_{l} \circ \mathbf{F}^{-1}, \quad l=1, \ldots, n,
$$


i.e. the $q$-difference operators constitute nonnegative unbounded self-adjoint operators with purely discrete spectra in $L^{2}(\mathbb{A}, \hat{\Delta} \mathrm{d} \xi)$ that are simultaneously diagonalized by the three-parameter (inverse) deformed hyperoctahedral $q$-Whittaker transform F (7.4a), 7.4b).

\section{SCATtERING}

In Ref. D4 the scattering operator for a wide class of quantum lattice models was determined by stationary-phase methods originating from Ref. [R4]. It follows from the diagonalization in Theorem 1 that our difference Toda chains fit within this class of lattice models. Indeed, the deformed hyperoctahedral $q$-Whittaker functions $p_{\lambda}, \lambda \in \Lambda$ belong to the family of orthogonal polynomials defined in D4, Sec. 2], since the orthogonality weight function $\hat{\Delta}(\xi)(3.4 \mathrm{~b})$ is of the indicated form (with $R=B C_{n}$ ) and moreover meets the demanded analyticity requirements. We will close by briefly indicating how the general scattering results from Ref. D4, Sec. 4.2] specialize in the present difference Toda setting.

Let $\mathcal{H}_{0}$ be the self-adjoint discrete Laplacian in $\ell^{2}(\Lambda)$ of the form

$$
\left(\mathcal{H}_{0} f\right)(\lambda):=\sum_{\substack{1 \leq j \leq n \\ \lambda+e_{j} \in \Lambda}} f\left(\lambda+e_{j}\right)+\sum_{\substack{1 \leq j \leq n \\ \lambda-e_{j} \in \Lambda}} f\left(\lambda-e_{j}\right) \quad\left(f \in \ell^{2}(\Lambda)\right),
$$

and let $\mathcal{H}$ denote the pushforward

$$
\mathcal{H}:=\boldsymbol{\Delta}^{1 / 2} H \boldsymbol{\Delta}^{-1 / 2}
$$

of the difference Toda Hamiltonian $H$ (4.7) onto the Hilbert space $\ell^{2}(\Lambda)$ via the Hilbert space isomorphism $\Delta^{1 / 2}: \ell^{2}\left(\rho_{0}+\Lambda, \Delta\right) \rightarrow \ell^{2}(\Lambda)$ given by

$$
\left(\boldsymbol{\Delta}^{1 / 2} f\right)(\lambda):=\Delta_{\lambda}^{1 / 2} f\left(\rho_{0}+\lambda\right) \quad\left(f \in \ell^{2}\left(\rho_{0}+\Lambda, \Delta\right)\right)
$$

(where $\boldsymbol{\Delta}^{-1 / 2}:=\left(\boldsymbol{\Delta}^{1 / 2}\right)^{-1}$ ). Clearly, one has by Theorem 1 that

$$
\mathcal{H}=\mathcal{F}^{-1} \hat{E} \mathcal{F} \quad \text { with } \quad \mathcal{F}:=\hat{\boldsymbol{\Delta}}^{1 / 2} \boldsymbol{F} \boldsymbol{\Delta}^{-1 / 2},
$$

where $\hat{\boldsymbol{\Delta}}^{1 / 2}: L^{2}(\mathbb{A}, \hat{\Delta} \mathrm{d} \xi) \rightarrow L^{2}(\mathbb{A})$ denotes the Hilbert space isomorphism given by

$$
\left(\hat{\Delta}^{1 / 2} \hat{f}\right)(\xi):=\hat{\Delta}^{1 / 2}(\xi) \hat{f}(\xi) \quad\left(\hat{f} \in L^{2}(\mathbb{A}, \hat{\Delta} \mathrm{d} \xi)\right)
$$

(and $\hat{E}(4.8 \mathrm{~b}$ ) is now regarded as a self-adjoint bounded multiplication operator in $\left.L^{2}(\mathbb{A})\right)$. Moreover, it is elementary that the spectral decomposition of the discrete Laplacian $\mathcal{H}_{0}$ is given by

$$
\mathcal{H}_{0}=\mathcal{F}_{0}^{-1} \hat{E} \mathcal{F}_{0}
$$

where $\mathcal{F}_{0}: \ell^{2}(\Lambda) \rightarrow L^{2}(\mathbb{A})$ denotes the Fourier isomorphism

$$
\left(\mathcal{F}_{0} f\right)(\xi):=\sum_{\lambda \in \Lambda} f(\lambda) \overline{\chi_{\xi}(\lambda)}
$$

$\left(f \in \ell^{2}(\Lambda)\right)$ with the inversion formula

$$
\left(\mathcal{F}_{0}^{-1} \hat{f}\right)(\lambda)=\int_{\mathbb{A}} \hat{f}(\xi) \chi_{\xi}(\lambda) \mathrm{d} \xi
$$

$\left(\hat{f} \in L^{2}(\mathbb{A})\right)$. Here we have employed the anti-invariant Fourier kernel

$$
\chi_{\xi}(\lambda):=\frac{1}{(2 \pi)^{n / 2} i^{n^{2}}} \sum_{w \in W} \operatorname{sign}(w) e^{i\langle w(\rho+\lambda), \xi\rangle},
$$


with $\operatorname{sign}(w)=\epsilon_{1} \cdots \epsilon_{n} \operatorname{sign}(\sigma)$ for $w=(\sigma, \epsilon) \in W=S_{n} \ltimes\{1,-1\}^{n}$ and $\rho=$ $(n, n-1, \ldots, 2,1)$. Notice that $\mathcal{F}_{0}$ is recovered from $\mathcal{F}$ in the limit $q \rightarrow 0, \hat{t}_{r} \rightarrow 0$ $(r=0, \ldots, 3)$.

The scattering operator describing the large-times asymptotics of the difference Toda dynamics $e^{i \mathcal{H} t}$ relative to the Laplacian's reference dynamics $e^{i \mathcal{H}_{0} t}$ turns out to be governed by an $n$-particle scattering matrix $\hat{\mathcal{S}}(\xi)$ that factorizes in two-particle pair matrices and one-particle boundary matrices:

$$
\hat{\mathcal{S}}(\xi):=\prod_{1 \leq j<k \leq n} s\left(\xi_{j}-\xi_{k}\right) s\left(\xi_{j}+\xi_{k}\right) \prod_{1 \leq j \leq n} s_{0}\left(\xi_{j}\right)
$$

with

$$
s(x):=\frac{\left(q e^{i x}\right)_{\infty}}{\left(q e^{-i x}\right)_{\infty}} \quad \text { and } \quad s_{0}(x):=\frac{\left(q e^{2 i x}\right)_{\infty}}{\left(q e^{-2 i x}\right)_{\infty}} \prod_{0 \leq r \leq 3} \frac{\left(\hat{t}_{r} e^{-i x}\right)_{\infty}}{\left(\hat{t}_{r} e^{i x}\right)_{\infty}} .
$$

To make the latter statement precise, let us denote by $C_{0}\left(\mathbb{A}_{\mathrm{reg}}\right)$ the dense subspace of $L^{2}(\mathbb{A})$ consisting of smooth test functions with compact support in the open dense subset $\mathbb{A}_{\text {reg }} \subset \mathbb{A}$ on which the components of the gradient

$$
\nabla \hat{E}(\xi)=\left(-2 \sin \left(\xi_{1}\right), \ldots,-2 \sin \left(\xi_{n}\right)\right), \quad \xi \in \mathbb{A}
$$

do not vanish and are all distinct in absolute value. We now define an unitary multiplication operator $\hat{\mathcal{S}}: L^{2}(\mathbb{A}, \mathrm{d} \xi) \rightarrow L^{2}(\mathbb{A}, \mathrm{d} \xi)$ via its restriction to $C_{0}\left(\mathbb{A}_{\text {reg }}\right)$ as follows:

$$
(\hat{\mathcal{S}} \hat{f})(\xi):=\hat{\mathcal{S}}\left(w_{\xi} \xi\right) \hat{f}(\xi) \quad\left(\hat{f} \in C_{0}\left(\mathbb{A}_{\mathrm{reg}}\right),\right.
$$

where $w_{\xi} \in W$ for $\xi \in \mathbb{A}_{\text {reg }}$ is such that the components of $w_{\xi} \nabla \hat{E}(\xi)$ are all positive and reordered from large to small.

Theorem 4.2 and Corollary 4.3 of Ref. D4 then provide the following explicit formulas for the wave operators and scattering operator of our difference Toda chain.

Theorem 5 (Wave and Scattering Operators). The operator limits

$$
\Omega^{ \pm}:=s-\lim _{t \rightarrow \pm \infty} e^{i t \mathcal{H}} e^{-i t \mathcal{H}_{0}}
$$

converge in the strong $\ell^{2}(\Lambda)$-norm topology and the corresponding wave operators $\Omega^{ \pm}$intertwining the difference Toda dynamics $e^{i \mathcal{H} t}$ with the discrete Laplacian's dynamics $e^{i \mathcal{H}_{0} t}$ are given by unitary operators in $\ell^{2}(\Lambda)$ of the form

$$
\Omega^{ \pm}=\mathcal{F}^{-1} \circ \hat{\mathcal{S}}^{\mp 1 / 2} \circ \mathcal{F}_{0}
$$

where the branches of the square roots are to be chosen such that

$$
s(x)^{1 / 2}=\frac{\left(q e^{i x}\right)_{\infty}}{\left|\left(q e^{i x}\right)_{\infty}\right|} \quad \text { and } \quad s_{0}(x)^{1 / 2}=\frac{\left(q e^{2 i x}\right)_{\infty}}{\left|\left(q e^{2 i x}\right)_{\infty}\right|} \prod_{0 \leq r \leq 3} \frac{\left|\left(\hat{t}_{r} e^{i x}\right)_{\infty}\right|}{\left(\hat{t}_{r} e^{i x}\right)_{\infty}} .
$$

Hence, the scattering operator relating the large-times asymptotics of the difference Toda dynamics $e^{i \mathcal{H} t}$ for $t \rightarrow-\infty$ and $t \rightarrow+\infty$ is given by the unitary operator

$$
\mathcal{S}:=\left(\Omega^{+}\right)^{-1} \Omega^{-}=\mathcal{F}_{0}^{-1} \circ \hat{\mathcal{S}} \circ \mathcal{F}_{0}
$$

The degenerate case of the difference Toda chain discussed in Section 7 is also covered by Theorem 5, upon setting $\rho_{0}$ equal to the nulvector in Eq. (8.2), replacing $H$ (4.7) by $\mathrm{H}(7.3)$ in $\mathcal{H}(8.1$ ) and $\boldsymbol{F}$ (4.6a), (4.6b) by $\mathbf{F}(7.4 \mathrm{a}),(17.4 \mathrm{~b})$ in $\mathcal{F}(8.3)$, and substituting $\hat{t}_{0}=0$ overall. 


\section{Appendix A. MACDONALD-KOORNWINDER POLYNOMIALS}

This appendix collects some key properties of the Macdonald-Koornwinder multivariate Askey-Wilson polynomials [K, D3, M]. In the case of one variable $(n=1)$, the properties below specialize to well-known formulas for the Askey-Wilson polynomials (see e.g. [KLS]

The Macdonald-Koornwinder polynomials $\mathbf{p}_{\lambda}(\xi)(\lambda \in \Lambda, \xi \in \mathbb{T})$ are defined as polynomials of the type in Eqs. (3.3a), 3.3b), (3.4a) associated with the weight function [K, Sec. 5], M, Ch. 5.3]:

$$
\hat{\boldsymbol{\Delta}}(\xi)=\frac{1}{(2 \pi)^{n}} \prod_{1 \leq j \leq n}\left|\frac{\left(e^{2 i \xi_{j}}\right)_{\infty}}{\prod_{0 \leq r \leq 3}\left(\hat{t}_{r} e^{i \xi_{j}}\right)_{\infty}}\right|^{2} \prod_{1 \leq j<k \leq n}\left|\frac{\left(e^{i\left(\xi_{j}+\xi_{k}\right)}, e^{i\left(\xi_{j}-\xi_{k}\right)}\right)_{\infty}}{\left(t e^{i\left(\xi_{j}+\xi_{k}\right)}, t e^{i\left(\xi_{j}-\xi_{k}\right)}\right)_{\infty}}\right|^{2}
$$

with $q \in(0,1)$ and $t, \hat{t}_{r} \in(-1,1) \backslash\{0\}(r=0, \ldots, 3)$. For $t \rightarrow 0$ this weight function passes into that of Eq. (3.4b), whence the polynomials in question degenerate in this limit continuously to the deformed hyperoctahedral $q$-Whittaker functions of Section 3. Notice in this respect that for $x \in \mathbb{R}$ and $|t|<\varepsilon(<1)$ quotients of the form $\left(e^{i x}\right)_{\infty} /\left(t e^{i x}\right)_{\infty}$ remain bounded in absolute value by $(-1)_{\infty} /(\varepsilon)_{\infty}$, so we may interchange limits and integration for $t \rightarrow 0$ when integrating trigonometric polynomials against the Macdonald-Koornwinder weight function $\hat{\boldsymbol{\Delta}}(\xi)$ over the bounded alcove $\mathbb{A}$ (by dominated convergence).

The normalized Macdonald-Koornwinder polynomials

$$
\mathbf{P}_{\lambda}(\xi):=\mathbf{c}_{\lambda} \mathbf{p}_{\lambda}(\xi) \quad\left(\lambda \in \Lambda_{n}\right)
$$

where

$$
\mathbf{c}_{\lambda}:=\prod_{1 \leq j \leq n} \frac{\left(\tau_{j}^{2}\right)_{2 \lambda_{j}}}{\prod_{0 \leq r \leq 3}\left(t_{r} \tau_{j}\right)_{\lambda_{j}}} \prod_{1 \leq j<k \leq n} \frac{\left(\tau_{j} \tau_{k}\right)_{\lambda_{j}+\lambda_{k}}}{\left(t \tau_{j} \tau_{k}\right)_{\lambda_{j}+\lambda_{k}}} \frac{\left(\tau_{j} \tau_{k}^{-1}\right)_{\lambda_{j}-\lambda_{k}}}{\left(t \tau_{j} \tau_{k}^{-1}\right)_{\lambda_{j}-\lambda_{k}}}
$$

with $\tau_{j}:=t^{n-j} t_{0}(j=1, \ldots, n)$ and $t_{r}(r=0, \ldots, 3)$ given by Eq. (4.1), satisfy the following orthogonality relations [K, Sec. 5], [D3, Sec. 7], [M, Ch. 5.3]:

$$
\int_{\mathbb{A}} \mathbf{P}_{\lambda}(\xi) \overline{\mathbf{P}_{\mu}(\xi)} \hat{\boldsymbol{\Delta}}(\xi) \mathrm{d} \xi= \begin{cases}\boldsymbol{\Delta}_{\lambda}^{-1} & \text { if } \lambda=\mu \\ 0 & \text { otherwise }\end{cases}
$$

with

$$
\begin{aligned}
\boldsymbol{\Delta}_{\lambda} & :=\boldsymbol{\Delta}_{0} \prod_{1 \leq j \leq n}\left(\frac{1-\tau_{j}^{2} q^{2 \lambda_{j}}}{1-\tau_{j}^{2}} \prod_{0 \leq r \leq 3} \frac{\left(t_{r} \tau_{j}\right)_{\lambda_{j}}}{\left(q t_{r}^{-1} \tau_{j}\right)_{\lambda_{j}}}\right) \\
& \times \prod_{1 \leq j<k \leq n} \frac{1-\tau_{j} \tau_{k} q^{\lambda_{j}+\lambda_{k}}}{1-\tau_{j} \tau_{k}} \frac{\left(t \tau_{j} \tau_{k}\right)_{\lambda_{j}+\lambda_{k}}}{\left(q t^{-1} \tau_{j} \tau_{k}\right)_{\lambda_{j}+\lambda_{k}}} \frac{1-\tau_{j} \tau_{k}^{-1} q^{\lambda_{j}-\lambda_{k}}}{1-\tau_{j} \tau_{k}^{-1}} \frac{\left(t \tau_{j} \tau_{k}^{-1}\right)_{\lambda_{j}-\lambda_{k}}}{\left(q t^{-1} \tau_{j} \tau_{k}^{-1}\right)_{\lambda_{j}-\lambda_{k}}}
\end{aligned}
$$

and

$$
\boldsymbol{\Delta}_{0}:=\prod_{1 \leq j \leq n} \frac{\left(q, t^{j}\right)_{\infty} \prod_{0 \leq r<s \leq 3}\left(\hat{t}_{r} \hat{t}_{s} t^{n-j}\right)_{\infty}}{\left(t, \hat{t}_{0} \hat{t}_{1} \hat{t}_{2} \hat{t}_{3} t^{2 n-j-1}\right)_{\infty}}
$$


These orthogonal polynomials satisfy moreover a second-order $q$-difference equation [K, Sec. 5], [M, Ch. 5.3, 4.4]:

$$
\begin{aligned}
& \mathbf{P}_{\lambda}(\xi) \sum_{j=1}^{n}\left(q^{-1} \hat{t}_{0} \hat{t}_{1} \hat{t}_{2} \hat{t}_{3} t^{2 n-1-j}\left(q^{\lambda_{j}}-1\right)+t^{j-1}\left(q^{-\lambda_{j}}-1\right)\right)= \\
& \sum_{1 \leq j \leq n} \hat{V}_{j}(\xi)\left(\mathbf{P}_{\lambda}\left(\xi-i \log (q) e_{j}\right)-\mathbf{P}_{\lambda}(\xi)\right)+\hat{V}_{j}(-\xi)\left(\mathbf{P}_{\lambda}\left(\xi+i \log (q) e_{j}\right)-\mathbf{P}_{\lambda}(\xi)\right),
\end{aligned}
$$

with

$$
\hat{V}_{j}(\xi):=\frac{\prod_{0 \leq r \leq 3}\left(1-\hat{t}_{r} e^{i \xi_{j}}\right)}{\left(1-e^{2 i \xi_{j}}\right)\left(1-q e^{2 i \xi_{j}}\right)} \prod_{\substack{1 \leq k \leq n \\ k \neq j}} \frac{1-t e^{i\left(\xi_{j}+\xi_{k}\right)}}{1-e^{i\left(\xi_{j}+\xi_{k}\right)}} \frac{1-t e^{i\left(\xi_{j}-\xi_{k}\right)}}{1-e^{i\left(\xi_{j}-\xi_{k}\right)}},
$$

and a Pieri-type recurrence formula [D3, Sec. 6], M, Ch. 5.3, 4.4]:

$$
\begin{aligned}
\mathbf{P}_{\lambda}(\xi) & \sum_{j=1}^{n}\left(2 \cos \left(\xi_{j}\right)-\hat{\tau}_{j}-\hat{\tau}_{j}^{-1}\right)= \\
& \sum_{\substack{1 \leq j \leq n \\
\lambda+e_{j} \in \Lambda}} V_{j}^{+}(\lambda)\left(\hat{\tau}_{j} \mathbf{P}_{\lambda+e_{j}}(\xi)-\mathbf{P}_{\lambda}(\xi)\right)+\sum_{\substack{1 \leq j \leq n \\
\lambda-e_{j} \in \Lambda}} V_{j}^{-}(\lambda)\left(\hat{\tau}_{j}^{-1} \mathbf{P}_{\lambda-e_{j}}(\xi)-\mathbf{P}_{\lambda}(\xi)\right),
\end{aligned}
$$

with $\hat{\tau}_{j}:=t^{n-j} \hat{t}_{0}(j=1, \ldots, n)$ and

$$
\begin{aligned}
& V_{j}^{+}(\lambda):=\frac{\hat{\tau}_{1}^{-1} \prod_{0 \leq r \leq 3}\left(1-t_{r} \tau_{j} q^{\lambda_{j}}\right)}{\left(1-\tau_{j}^{2} q^{2 \lambda_{j}}\right)\left(1-\tau_{j}^{2} q^{2 \lambda_{j}+1}\right)} \prod_{\substack{1 \leq k \leq n \\
k \neq j}} \frac{1-t \tau_{j} \tau_{k} q^{\lambda_{j}+\lambda_{k}}}{1-\tau_{j} \tau_{k} q^{\lambda_{j}+\lambda_{k}}} \frac{1-t \tau_{j} \tau_{k}^{-1} q^{\lambda_{j}-\lambda_{k}}}{1-\tau_{j} \tau_{k}^{-1} q^{\lambda_{j}-\lambda_{k}}}, \\
& V_{j}^{-}(\lambda):=\frac{\hat{\tau}_{1} \prod_{0 \leq r \leq 3}\left(1-t_{r}^{-1} \tau_{j} q^{\lambda_{j}}\right)}{\left(1-\tau_{j}^{2} q^{2 \lambda_{j}}\right)\left(1-\tau_{j}^{2} q^{2 \lambda_{j}-1}\right)} \prod_{\substack{1 \leq k \leq n \\
k \neq j}} \frac{1-t^{-1} \tau_{j} \tau_{k} q^{\lambda_{j}+\lambda_{k}}}{1-\tau_{j} \tau_{k} q^{\lambda_{j}+\lambda_{k}}} \frac{1-t^{-1} \tau_{j} \tau_{k}^{-1} q^{\lambda_{j}-\lambda_{k}}}{1-\tau_{j} \tau_{k}^{-1} q^{\lambda_{j}-\lambda_{k}}}
\end{aligned}
$$

(where the vectors $e_{1}, \ldots, e_{n}$ refer to the standard unit basis of $\mathbb{R}^{n}$ ).

\section{REFERENCES}

B. O. Babelon, Equations in dual variables for Whittaker functions, Lett. Math. Phys. 65 (2003), 229-240.

BC. A. Borodin and I. Corwin, Macdonald processes, Probab. Theory Related Fields 158 (2014), 225-400.

C. I. Cherednik, Whittaker limits of difference spherical functions, Int. Math. Res. Not. IMRN 2009, 3793-3842.

D1. J.F. van Diejen, Deformations of Calogero-Moser systems and finite Toda chains, Theoret. and Math. Phys. 99 (1994), 549-554.

D2. _ Difference Calogero-Moser systems and finite Toda chains, J. Math. Phys. 36 (1995), 1299-1323.

D3. _ Properties of some families of hypergeometric orthogonal polynomials in several variables, Trans. Amer. Math. Soc. 351 (1999), 233-270.

D4. L Scattering theory of discrete (pseudo) Laplacians on a Weyl chamber, Amer. J. Math. 127 (2005), 421-458.

DG. J.J. Duistermaat and F.A. Grünbaum, Differential equations in the spectral parameter, Comm. Math. Phys. 103 (1986), 177-240.

E. P. Etingof, Whittaker functions on quantum groups and $q$-deformed Toda operators, in: Differential Topology, Infinite-Dimensional Lie Algebras, and Applications, A. Astashkevich and S. Tabachnikov (eds.), Amer. Math. Soc. Transl. Ser. 2, Vol.194, Amer. Math. Soc., Providence, RI, 1999, p. 9-25. 
F. L. Fehér, Action-angle map and duality for the open Toda lattice in the perspective of Hamiltonian reduction, Phys. Lett. A 377 (2013), 2917-2921.

GLO1. A. Gerasimov, D. Lebedev, and S. Oblezin, On $q$-deformed $g l_{l+1}$-Whittaker function III, Lett. Math. Phys. 97 (2011), 1-24.

GLO2. - Quantum Toda chains intertwined, St. Petersburg Math. J. 22 (2011), 411-435.

GW. R. Goodman and N.R. Wallach, Classical and Quantum Mechanical Systems of TodaLattice Type III. Joint Eigenfunctions of the Quantized Systems, Commun. Math. Phys. 105 (1986), 473-509.

G. F.A. Grünbaum, The bispectral problem: an overview, in: Special Functions 2000: Current Perspective and Future Directions, J. Bustoz, M.E.H. Ismail, and S.K. Suslov (eds.), NATO Sci. Ser. II Math. Phys. Chem., Vol. 30, Kluwer Acad. Publ., Dordrecht, 2001, p. 129-140.

HR. M. Hallnäs and S.N.M. Ruijsenaars, Kernel functions and Bäcklund transformations for relativistic Calogero-Moser and Toda systems, J. Math. Phys. 53 (2012), 123512.

I. V.I. Inozemtsev, The finite Toda lattices, Comm. Math. Phys. 121 (1989), 629-638.

KLS. R. Koekoek, P. Lesky, and R.F. Swarttouw, Hypergeometric Orthogonal Polynomials and their q-Analogues, Springer Monographs in Mathematics, Springer Verlag, New York, 2010.

K. T.H. Koornwinder, Askey-Wilson polynomials for root systems of type $B C$, in: Hypergeometric Functions on Domains of Positivity, Jack Polynomials, and Applications (D. St. P. Richards, ed.), Contemp. Math., Vol. 138, Amer. Math. Soc., Providence, RI, 1992, p. 189-204.

Ko. B. Kostant, Quantization and representation theory, in: Representation Theory of Lie groups (G.L. Luke, ed.), London Mathematical Society Lecture Note Series, Vol. 34, Cambridge University Press, Cambridge-New York, 1979, p. 287-316.

Kz. K.K. Kozlowski, Aspects of the inverse problem for the Toda chain, J. Math. Phys. 54 (2013), 121902.

KJC. V.B. Kuznetsov, M.F. Jørgensen, and P.L. Christiansen, New boundary conditions for integrable lattices, J. Phys. A 28 (1995), 4639-4654.

KT. V.B. Kuznetsov and A.V. Tsyganov, Quantum relativistic Toda chains, J. Math. Sci. 80 (1996), 1802-1810.

M. I.G. Macdonald, Affine Hecke Algebras and Orthogonal Polynomials, Cambridge University Press, Cambridge, 2003.

O. T. Oshima, Completely integrable systems associated with classical root systems, SIGMA Symmetry Integrability 3 (2007), 061.

R1. S.N.M. Ruijsenaars, Relativistic Toda systems, Comm. Math. Phys. 133 (1990), 217-247.

R2. Finite-dimensional soliton systems, in: Integrable and Superintegrable Systems, B. Kupershmidt (ed.), World Sci. Publ., Teaneck, NJ, 1990, p. 165-206.

R3. Systems of Calogero-Moser type. Particles and fields, in: Particles and Fields (Banff, 1994), G. Semenoff, L. Vinet (eds.), CRM Ser. Math. Phys., Springer Verlag, New York, 1999, p. 251-352.

R4. 는 Factorized weight functions vs. factorized scattering. Comm. Math. Phys. 228 (2002), 467-494.

S. A. Sevostyanov, Quantum deformation of Whittaker modules and the Toda lattice, Duke Math. J. 105 (2000), 211-238.

Sh. N. Shimeno, A limit transition from Heckman-Opdam hypergeometric functions to the Whittaker functions associated with root systems, arXiv.0812.3773.

Sk1. E.K. Sklyanin, Boundary conditions for integrable quantum systems, J. Phys. A 21 (1988), 2375-2389.

Sk2. Mispectrality for the quantum open Toda chain, J. Phys. A 46 (2013), 382001.

Su1. Y.B. Suris, Discrete time generalized Toda lattices: complete integrability and relation with relativistic Toda lattices, Phys. Lett. A 145 (1990), 113-119.

Su2. The Problem of Integrable Discretization: Hamiltonian Approach, Progress in Math., Vol. 219, Birkäuser Verlag, Basel, 2003.

St. B. Sutherland, An introduction to the Bethe ansatz, in: Exactly Solvable Problems in Condensed Matter and Relativistic Field Theory (Panchgani, 1985), B.S. Shastry, S.S. Jha and V. Singh (eds.), Lecture Notes in Phys., Vol. 242, Springer Verlag, Berlin, 1985, p. $1-95$. 
Instituto de Matemática y Física, Universidad de Talca, Casilla 747, Talca, Chile

E-mail address: diejen@inst-mat.utalca.cl

Facultad de Matemáticas, Pontificia Universidad Católica de Chile, Casilla 306, Correo 22, Santiago, Chile

E-mail address: eemsiz@mat.puc.cl 